\title{
XMM-Newton Observations of Two High-Redshift Quasars: RX J1028-0844 and BR 0351-1034
}

\section{Citation}

Grupe, D., S. Mathur, B. Wilkes, and M. Elvis. 2004. “XMM-Newton Observations of Two HighRedshift Quasars: RX J1028-0844 and BR 0351-1034." The Astronomical Journal 127 (1) (January): 1-9. doi:10.1086/380615.

\section{Published Version}

doi:10.1086/380615

\section{Permanent link}

http://nrs.harvard.edu/urn-3:HUL.InstRepos:30238400

\section{Terms of Use}

This article was downloaded from Harvard University's DASH repository, and is made available under the terms and conditions applicable to Other Posted Material, as set forth at http:// nrs.harvard.edu/urn-3:HUL.InstRepos:dash.current.terms-of-use\#LAA

\section{Share Your Story}

The Harvard community has made this article openly available.

Please share how this access benefits you. Submit a story.

Accessibility 
The Astronomical Journal, 127:1-9, 2004 January

(C) 2004. The American Astronomical Society. All rights reserved. Printed in U.S.A.

\title{
XMM-NEWTON OBSERVATIONS OF TWO HIGH-REDSHIFT QUASARS: RX J1028-0844 AND BR 0351-1034 ${ }^{1}$
}

\author{
D. Grupe and S. Mathur \\ Department of Astronomy, Ohio State University, 140 West 18th Avenue, Columbus, OH 43210; dgrupe@astronomy.ohio-state.edu, smita@astronomy.ohio-state.edu \\ AND \\ B. Wilkes AND M. Elvis \\ Harvard-Smithsonian Center for Astrophysics, 60 Garden Street, Cambridge, MA 02138 \\ Received 2003 August 20; accepted 2003 October 7
}

\begin{abstract}
We report the results of XMM-Newton observations of two high-redshift quasars, one radio-loud, RX J1028.6-0844 $(z=4.276)$ and one radio-quiet, BR 0351-1034 $(z=4.351)$. We find that the evidence for strong excess absorption toward RX $\mathrm{J} 1028-0844$ is marginal at best, contrary to previous claims. The superior sensitivity and broader, softer energy range of XMM-Newton $(0.2-10 \mathrm{keV})$ allows better determination of spectral parameters than much deeper $A S C A$ observations $(0.8-7 \mathrm{keV})$. Our XMM-Newton observations call into question several other $A S C A$ results of strong absorption toward high-redshift radio-loud quasars. RX J1028.6-0844 occupies the same parameter space in broadband spectral properties as the low-redshift BL Lac objects, showing no obvious evolution with redshift. The radio-quiet quasar BR 0351-1024 became fainter between ROSAT and $X M M$ observations by a factor of at least 5 , but with the present data we cannot determine whether there is an associated spectral change. These observations do not support previous claims of weaker X-ray emission from high-redshift, radio-quiet quasars. The soft X-ray spectral slope required to reconstruct the ROSAT PSPC hardness ratio of BR 0351-1034 is about $\alpha_{\mathrm{X}}=3.5$, the steepest X-ray slope ever observed in a high-redshift quasar and similar to that of low-redshift narrow-line Seyfert 1 galaxies.
\end{abstract}

Key words: galaxies: active — quasars: general — quasars: individual (BR 0351-1034, RX J1028-0844)

\section{INTRODUCTION}

High-redshift quasars are interesting not only for their record-setting quality but also because they can tell us about the formation of quasars and about conditions in the first few percent of the age of the universe. They allow us to study the evolution of a quasar's central engine (e.g., Vignali et al. 2001), the star formation in the early universe (e.g., Dietrich et al. 2002), and the intergalactic medium between the highredshift quasar and us (e.g., Péroux et al. 2001). Prior to ROSAT (Trümper 1982) only one quasar with $z>4.0$ was detected in X-rays, GB 1508+5714 ( $z=4.30$; Mathur \& Elvis 1995). Only one high-redshift quasar was discovered during the ROSAT AllSky Survey (RASS; Voges et al. 1999), RX J1028.6-0844 (Zickgraf et al. 1997). The first X-ray-selected, high-redshift quasar was RX J1759.4+6632 ( $z=4.320$; Henry et al. 1994), found in a deep ROSAT Position Sensitive Proportional Counter (PSPC; Pfeffermann et al. 1987) observation. Other sources were detected in X-rays but selected in other wavelength bands, typically by their radio emission, e.g., GB 1428+4217 $(z=4.72$; Boller et al. 2000) or at optical wavelengths (e.g., Q0000-263, $z=4.111$; Bechtold et al. 1994). Thanks to the Sloan Digital Sky Survey (SDSS; York et al. 2000) the number of high-redshift quasars, even to redshifts $z>6$, has increased dramatically, and several of them have been detected in X-rays (e.g., Mathur, Wilkes, \& Ghosh 2002; Brandt et al. 2002; Vignali et al. 2003b). ${ }^{2}$

\footnotetext{
${ }^{1}$ Based on observations with XMM-Newton, an ESA science mission with instruments and contributions directly funded by ESA member states and NASA.

${ }^{2}$ A complete list of $z>4$ quasars with $\mathrm{X}$-ray detections is given at http://www.astro.psu.edu/users/niel/papers/highz-xray-detected.dat.
}

While detection of these $z>4$ quasars in X-rays has opened up a new field of research, the results are conflicting. For example, Brinkmann, Yuan, \& Siebert (1997b) and Bechtold et al. (2001) find that high-redshift quasars are more X-ray quiet and have flatter X-ray spectra than the low-z quasars, in contradiction to the Mathur et al. (2002) results. Vignali et al. (2003b) also advocated the view that high-redshift quasars are more X-ray weak, but from a larger sample Vignali, Brandt, \& Schneider (2003a) concluded that the trend is with luminosity rather than redshift. One main reason behind these contradictory results is that they have all been based on short, snapshot observations. The resulting total counts, on the order of a few tens, are generally too few to permit spectral analysis. As a result, derived quantities such as $\alpha_{\text {ox }}{ }^{3}$ have a strong dependence on the underlying assumptions of spectral shape and absorbing column density. To understand high-redshift quasars and to compare them to their low-redshift cousins, we have initiated a program to obtain X-ray spectra of highredshift quasars using XMM-Newton. The sample consists of both radio-loud and radio-quiet quasars to probe differential evolution between the two classes, if any. Here we present results of the AO1 observations of a radio-loud quasar RX J1028-0844 and a radio-quiet quasar BR 0351-1034.

The high-redshift quasar RX J1028.6-0844 (RASS position: $\alpha=10^{\mathrm{h}} 28^{\mathrm{m}} 38^{\mathrm{s}} .9 ; \delta=-08^{\circ} 44^{\prime} 29^{\prime \prime}$ [J2000.0]; $z=4.276$ ) was identified by Zickgraf et al. (1997) in an identification program of northern X-ray sources (Appenzeller et al. 1998) detected during the RASS. Its PSPC count rate during the RASS was $0.035 \pm 0.011$ counts $\mathrm{s}^{-1}$, which transfers to a rest-

\footnotetext{
${ }^{3}$ The X-ray loudness is defined by Tananbaum et al. (1979) as $\alpha_{\text {ox }}=-0.384 \log \left(f_{2 \mathrm{keV}} / f_{2500} \AA\right)$.
} 
frame $2-10 \mathrm{keV}$ luminosity $L_{2-10 \mathrm{keV}}=6.4 \times 10^{46} \mathrm{ergs} \mathrm{s}^{-1}$, which makes it one of the most X-ray luminous sources in the universe (Zickgraf et al. 1997). RX J1028.6-0844 is associated with a close by radio source PKS B1026-084 with a flux of $220 \mathrm{mJy}$ at $5 \mathrm{GHz}$ in the Parkes radio survey (Otrupcek \& Wright 1991). From its extreme luminosities at all wavelengths and its radio loudness RX J1028.6-084 is considered a BL Lac object (Yuan et al. 2000). In a long $67 \mathrm{hr}$ observation by $A S C A$ (Tanaka, Inoue, \& Holt 1994), Yuan et al. (2000) found evidence of very high neutral absorption at the rest-frame of RX J1028.6-0844. Assuming solar abundances, an absorption column of $2 \times 10^{23} \mathrm{~cm}^{-2}$ was found. However, it was not clear from the $A S C A$ data whether this absorption is associated with the quasar or if it is related to a damped Ly $\alpha$ absorber at $z=3.42$ (Péroux et al. 2001).

The high-redshift, radio-quiet quasar BR 0351-1034 ( $\alpha=$ $03^{\mathrm{h}} 53^{\mathrm{m}} 46^{\mathrm{s}} .9, \delta=-10^{\circ} 25^{\prime} 19^{\prime \prime} .0[\mathrm{~J} 2000.0] ; z=4.351$ ) was discovered by the APM high-redshift quasar survey by Irwin, McMahon, \& Hazard (1991). Storrie-Lombardi et al. (1996a) reported that BR 0351-1034 was one of the most unusual sources of their survey of high-redshift APM quasars with intervening absorption systems. They found saturated C IV absorption and a large number of absorption lines associated with damped Ly $\alpha$ absorption systems at $z=3.633,4.098$, and 4.351. The source was first detected in X-rays by ROSAT in a 9.1 ks pointed PSPC observation with $54 \pm 13$ counts (Kaspi, Brandt, \& Schneider 2000).

In this paper we present the results of the XMM-Newton (Jansen et al. 2001) observations of these two quasars. The short (5 ks) observation of RX J1028.6-0844 was planned before the long $A S C A$ observation. The supreme sensitivity of the EPIC PN detector (Strüder et al. 2001) at soft X-rays and recent calibration efforts, allow measurements down to $0.2 \mathrm{keV}$ (or even less, Haberl et al. 2003), putting better constrains on the intrinsic absorption of the source than from previous X-ray missions. Our 26 ks observation of BR 0351-1034 was severely affected by the high background radiation. As a result, the spectral quality of the source was significantly compromised, and so the spectral parameters are not well constrained.

The paper is organized as follows: in $\S 2$ we describe the observations and data reduction, in $\S 3$ we present the results of the X-ray observation, which will be discussed in $\S 4$.

Throughout the paper spectral indices are energy spectral indices with $F_{\nu} \propto \nu^{-\alpha}$. Luminosities are calculated assuming a $\Lambda$ CDM cosmology with $\Omega_{M}=0.3, \Omega_{\Lambda}=0.7$, and a Hubble constant of $H_{0}=75 \mathrm{~km} \mathrm{~s}^{-1} \mathrm{Mpc}^{-1}$, using the formulae given to derive the luminosity distances given by Hogg (1999). All errors are $1 \sigma$ unless stated otherwise.

\section{OBSERVATIONS AND DATA ANALYSIS}

\subsection{Radio-loud Quasar RX J1028.6-0844}

RX J1028.6-0844 was observed by XMM-Newton on 2002 May 15 for a total of $5 \mathrm{ks}$ with the EPIC PN (Strüder et al. 2001) and $7.3 \mathrm{ks}$ with the EPIC MOS (Turner et al. 2001) detectors using thin filters. A high background flare was present during a short time of the observation. We excluded this time by creating a good time interval (GTI) file accepting only times when the background count rate of photons with energies greater than $10 \mathrm{keV}$ was less than 10 counts $\mathrm{s}^{-1}$. Only the EPIC PN observation was significantly affected by the flare. The GTI screening of the PN data results in a total observing time of $4350 \mathrm{~s}$. Source photons in the EPIC PN were collected in a circle with a radius of $35^{\prime \prime}$ and the background photons in a circle of a radius of $60^{\prime \prime}$ close by. The source photons in the MOS detectors were selected in a circle with a radius of $31^{\prime \prime} .25$ and the background from an annulus of $35^{\prime \prime}$ inner radius and $75^{\prime \prime}$ outer radius. We selected single and double events (PATTERN $\leq 4$ ) for the PN and single, double, triple, and quadruple events $($ PATTERN $\leq 12)$ for the MOS, for which the detectors are calibrated.

In addition to the XMM-Newton data of RX J1028.6-0844 we also retrieved the $A S C A$ data from observation of 1999 November 25 (seq-ID: 77011000; Yuan et al. 2000) from the $A S C A$ data archive at Goddard Space Flight Center in order to fit our XMM-Newton data together with the $A S C A$ data. Because of the decrease in efficiency below $1 \mathrm{keV}$ of the $A S C A$ Solid-State Imaging Spectrometers (SIS) after late $1994^{4}$ we considered only photons with $E>0.8 \mathrm{keV}$ for our analysis.

\subsection{Radio-quiet Quasar BR 0351-1034}

BR 0351-1034 was observed by XMM-Newton on 2002 August 23 for a total of $26.0 \mathrm{ks}$ with the EPIC PN and $27.7 \mathrm{ks}$ with the EPIC MOS detectors using thin filters. Due to a high background radiation during the last half of the observation, these data were unusable. The data were screened to create a GTI file with background count rate of photons with energies greater than $10 \mathrm{keV}$ to be less than 10 counts $\mathrm{s}^{-1}$. This screening results in total observing times of 15.8 and $19.5 \mathrm{ks}$ with the PN and MOS detectors, respectively.

Source counts were collected in a circle with a radius of $15^{\prime \prime}$ and the background from a nearby circular region with a radius of $30^{\prime \prime}$. Because of its small number of counts ( $\left.\S 3.2 .1\right)$ only the EPIC PN data with single and double events (PATTERN $\leq 4$ ) were used for the spectral analysis. Because of the faintness of the source, the results may be affected by the choice of the background region. We determined background from another nearby region as well and found the results with the two different backgrounds to be consistent with one another within errors.

The source was observed in a pointed ROSAT PSPC observation on 1992 January 27 (Obs-ID rp700531; Kaspi et al. 2000) for a total of $9.4 \mathrm{ks}$. We retrieved the data from the ROSAT Archive at MPE Garching and reanalyzed them. The source photons were collected in a circle with $R=75^{\prime \prime}$. Since the source is faint, again we selected two background regions, one in a circle with $R=150^{\prime \prime}$ near the source and a second one in an annulus around the source with an inner radius $R=100^{\prime \prime}$ and an outer radius $R=200^{\prime \prime}$. Again, we found that the choice of the background did not affect our results.

For both the sources, the XMM-Newton data were reduced using the XMM-Newton Science Analysis Software (XMMSAS), version 5.3.3, and the X-ray spectra were analyzed using XSPEC 11.2.0. The spectra were grouped by GRPPHA 3.0.0 in bins of at least 20 counts per bin. For both sources, the ancillary response matrix and the detector response matrix were created by the XMMSAS tasks "arfgen" and "rmfgen." The ASCA spectral data of RX J1028.6-0844 were selected by XSELECT, grouped by GRPPHA as the XMM-Newton data, and analyzed by XSPEC. The ROSAT data of BR 0351-1034 were analyzed using the Extended X-Ray Scientific Analysis System (EXSAS; Zimmermann et al. 1998), version 01APR. ${ }^{5}$ For the count-rate

\footnotetext{
${ }^{4}$ See the $A S C A$ Web site at http://heasarc.gsfc.nasa.gov/docs/asca/watchout .html.

${ }^{5}$ See http://wave.xray.mpe.mpg.de/exsas/users-guide.
} 
conversions between different X-ray missions, PIMMS 3.2 was used.

\section{RESULTS}

\section{1. $R X J 1028.6-0844$}

\subsubsection{The XMM-Newton Observation}

The mean count rates measured for the EPIC PN, MOS-1, and MOS-2 were $0.416 \pm 0.010,0.115 \pm 0.004$, and $0.094 \pm 0.005$ counts $s^{-1}$, respectively. On the short timescale of the observation (the 5 ks EPIC PN observation converts to $\approx 1 \mathrm{ks}$ in the object's rest frame) we could not detect any significant variability. We converted the EPIC PN count rate into ROSAT PSPC and $A S C A$ SIS count rates by using PIMMS and could not detect any significant changes in the count rate on long timescales either. None of the observations so far have shown any significant variability in this source. Yuan et al. (2000) discussed the temporal analysis of their $67 \mathrm{hr} A S C A$ observation and also found no significant variability.

Table 1 summarizes the results of a simple power-law fit with "cold" absorption of neutral elements at $z=0$ to the $X M M$ Newton data of RX J1028.6-0844. The fits to all instruments separately agree within the errors, except for the MOS-1, which shows a slightly higher absorption column than the other detectors. This latter is driven by one data point at about $0.7 \mathrm{keV}$ (see Fig. 1), so the discrepancy does not appear to be significant. The X-ray slope is about $\alpha_{\mathrm{X}}=0.3$. This is in good agreement with what has been found for other high-redshift, radio-loud quasars (e.g., Ferrero \& Brinkmann 2003). When data from all three $X M M$-Newton detectors are fitted together with absorption fixed at the Galactic value $\left(N_{\mathrm{H}}=4.59 \times 10^{20} \mathrm{~cm}^{-2}\right.$; Dickey \& Lockman 1990), the fit is good with $\chi^{2}=169$ for 160 degrees of freedom (dof; see Table 1 and Fig. 2). If the absorbing column density is left as a free parameter, the fit yields $N_{\mathrm{H}}=7 \pm 1 \times 10^{20} \mathrm{~cm}^{-2}$ and $\chi^{2}=174.6(161 \mathrm{dof})$. Thus, the best-fit $N_{\mathrm{H}}$ is consistent with the Galactic value, within errors, and the spectra do not show any compelling evidence of excess absorption.

\subsubsection{Comparison with ASCA Spectra}

The above result contradicts earlier $A S C A$ results, reported by Yuan et al. (2000), who found strong excess absorption toward the source. To quantify the difference and to verify the $A S C A$ results we fitted the $X M M-N e w t o n$ and $A S C A$ data with a power law plus Galactic absorption (fixed to the Galactic value) and intrinsic redshifted neutral absorption at the redshift of the quasar. We used the XSPEC model "zvfeabs" for the redshifted intrinsic absorption. The metal abundance $Z / Z_{\odot}$ was set to unity (solar abundance). The results of these fits are given in Table 2 . Here the $A S C A$ SIS detectors show a very high intrinsic column density of $N_{\mathrm{H}, \text { intr }}=15.2 \pm 14.0 \times 10^{22} \mathrm{~cm}^{-2}$, which confirms the results found by Yuan et al. (2000). However, fits to the PN data result in much lower column densities, as discussed above. Higher column densities are obtained with MOS detectors, which do not have well-calibrated response below $0.5 \mathrm{keV}$, and even higher column densities are found with $A S C A$ SIS detectors, which have no response below $0.8 \mathrm{keV}$. Thus, the inferred high column density appears to be a direct result of lack of low-energy response of these detectors.

To better understand the difference between XMM-Newton and $A S C A$ results, we performed the following additional tests. First, we fitted the data by fixing the intrinsic column density and X-ray slopes to the ones found by $A S C A$ $\left(N_{\mathrm{H}, \text { intr }}=21.1 \times 10^{22} \mathrm{~cm}^{-2}, \alpha_{\mathrm{X}}=0.72\right.$; Yuan et al. 2000). In the $0.2-1.0 \mathrm{keV}$ range we can clearly see strong deviations at energies below $0.8 \mathrm{keV}$ (Fig. 3). However, at energies above $0.8 \mathrm{keV}$ the data agree very well with the values found from the fits to the $A S C A$ data. Using the XMM-Newton and $A S C A$ data only in the $A S C A$ SIS $0.8-6.5 \mathrm{keV}$ energy range results in much higher column densities than when the whole detector energy ranges are used (Table 2). The data in the 0.8-6.5 energy range of the XMM-Newton detectors are indeed more or less consistent with the parameters found by $A S C A$. Clearly, the lack of

TABLE 1

Spectral Fit Parameters of RX J1028.6-0844 for a Power-Law Model with Neutral Absorption at $z=0$

\begin{tabular}{|c|c|c|c|}
\hline Detector & $\begin{array}{c}N_{\mathrm{H}} \\
\left(10^{22} \mathrm{~cm}^{-2}\right)\end{array}$ & $\alpha_{\mathrm{X}}$ & $\begin{array}{c}\chi^{2} \\
(\mathrm{dof})\end{array}$ \\
\hline \multirow[t]{2}{*}{$\mathrm{PN}^{1}$} & $0.0665 \pm 0.0128$ & $0.307 \pm 0.054$ & $91.8(84)$ \\
\hline & 0.0459 (fixed) & $0.233 \pm 0.032$ & $95.2(85)$ \\
\hline \multirow[t]{2}{*}{$\operatorname{MOS}-1^{2}}$. & $0.1653 \pm 0.0486$ & $0.424 \pm 0.110$ & $40.3(35)$ \\
\hline & 0.0459 (fixed) & $0.194 \pm 0.595$ & $47.0(36)$ \\
\hline \multirow[t]{2}{*}{ MOS-2 ${ }^{2}$} & $0.0883 \pm 0.0451$ & $0.314 \pm 0.112$ & $31.5(37)$ \\
\hline & 0.0459 (fixed) & $0.225 \pm 0.060$ & $32.4(38)$ \\
\hline \multirow[t]{2}{*}{ MOS- $1^{2}$ and MOS $-2^{2} \ldots \ldots \ldots \ldots \ldots \ldots$} & $0.1251 \pm 0.0332$ & $0.368 \pm 0.079$ & $73.0(74)$ \\
\hline & 0.0459 (fixed) & $0.209 \pm 0.042$ & $79.4(75)$ \\
\hline \multirow[t]{2}{*}{$A S C A$ SIS- $-0^{3}$ and SIS- $1^{4}$. } & $0.0611 \pm 0.1465$ & $0.416 \pm 0.207$ & $26.3(28)$ \\
\hline & 0.0459 (fixed) & $0.397 \pm 0.100$ & $26.5(29)$ \\
\hline \multirow[t]{2}{*}{$\mathrm{PN}^{1}+\mathrm{MOS}-1^{2}$ and MOS $-2^{2}$.. } & $0.0712 \pm 0.0119$ & $0.298 \pm 0.042$ & $169.0(160)$ \\
\hline & 0.0459 (fixed) & $0.225 \pm 0.025$ & $174.6(161)$ \\
\hline \multirow[t]{2}{*}{$\mathrm{PN}^{1}+A S C A$ SIS- $-0^{3}$ and SIS- $1^{4} \ldots \ldots$} & $0.0715 \pm 0.0122$ & $0.331 \pm 0.047$ & $119.0(113)$ \\
\hline & 0.0459 (fixed) & $0.249 \pm 0.031$ & $124.5(114)$ \\
\hline \multicolumn{4}{|l|}{$\mathrm{PN}^{1}+\mathrm{MOS}^{2} 1^{2}$ and MOS- $2^{2}+$} \\
\hline \multirow[t]{2}{*}{$A S C A$ SIS- $0^{3}$ and SIS- $1^{4}$} & $0.0725 \pm 0.0114$ & $0.304 \pm 0.040$ & $211.8(189)$ \\
\hline & 0.0459 (fixed) & $0.229 \pm 0.025$ & $206.0(190)$ \\
\hline
\end{tabular}

${ }^{1}$ Energy range $0.2-10 \mathrm{keV}$.

${ }^{2}$ Energy range $0.5-7.5 \mathrm{keV}$.

${ }^{3}$ Energy range $0.8-7.0 \mathrm{keV}$.

${ }^{4}$ Energy range $0.8-6.5 \mathrm{keV}$. 

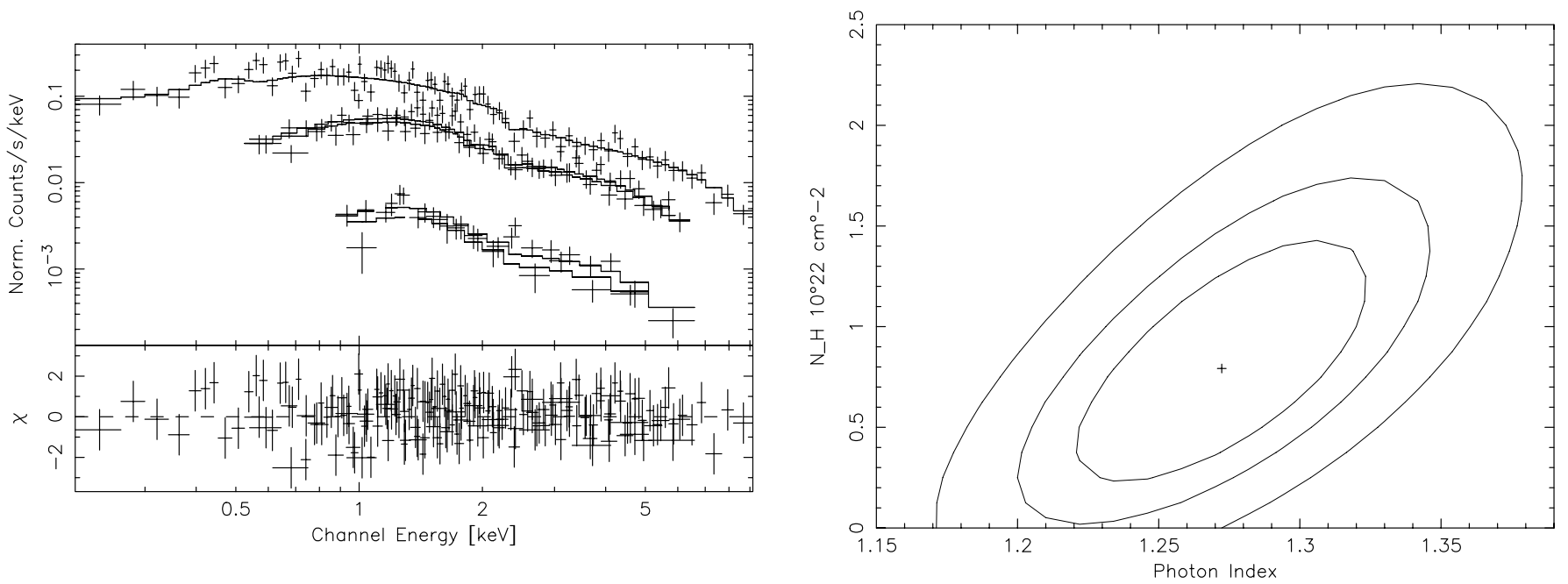

FIg. 1.-Power-law fit with neutral Galactic absorption (fixed to galactic value) and intrinsic absorption with metal abundance = solar to the EPIC PN, MOS-1 and MOS-2, and ASCA SIS-0 and SIS-1 data of RX J1028.6-0844 $\left(\alpha_{\mathrm{X}}=0.272, N_{\mathrm{H}, \mathrm{intr}}=0.79 \times 10^{22} \mathrm{~cm}^{-2}\right.$, Table 2). Left: Fits to the spectra of the EPIC PN (top) MOS (middle), and ASCA SIS (bottom) detectors. Right: 68\%, 90\%, and 99\% confidence levels of the column density of the intrinsic absorber vs. the photon index (photon index $\Gamma=\alpha_{\mathrm{X}}+1$; Table 2).

low-energy response of $A S C A$ SIS gives incorrect results, and the superior sensitivity of XMM-Newton in the soft energy band down to $0.2 \mathrm{keV}$ allows us to make accurate measurements of the absorbing column density.

Figure 1 displays the result of a power-law with Galactic and intrinsic absorption simultaneously fitted to the $X M M$ Newton PN, and MOS- 1 and MOS-2, and ASCA SIS-0 and SIS-1 data (Table 2). All the data including the $A S C A$ SIS data are well fitted with an X-ray spectral slope $\alpha_{\mathrm{X}}=0.3$ and an intrinsic absorption column $N_{\mathrm{H} \text {,intr }} \approx 10^{22} \mathrm{~cm}^{-2}$ with solar metal abundance, an order of magnitude lower than the column density based on $A S C A$ data alone.

To summarize, the $X M M$-Newton spectra do not show strong evidence of excess absorption toward RX J1028-0844. If there is excess absorption at all, and if it is at the redshift of the quasar, then it is an order of magnitude lower than that inferred from $A S C A$ data.

\subsubsection{Broadband Properties}

RX J1028.6-0844 is associated with a nearby radio source PKS B1026-084 within the RASS error circle (Zickgraf et al.

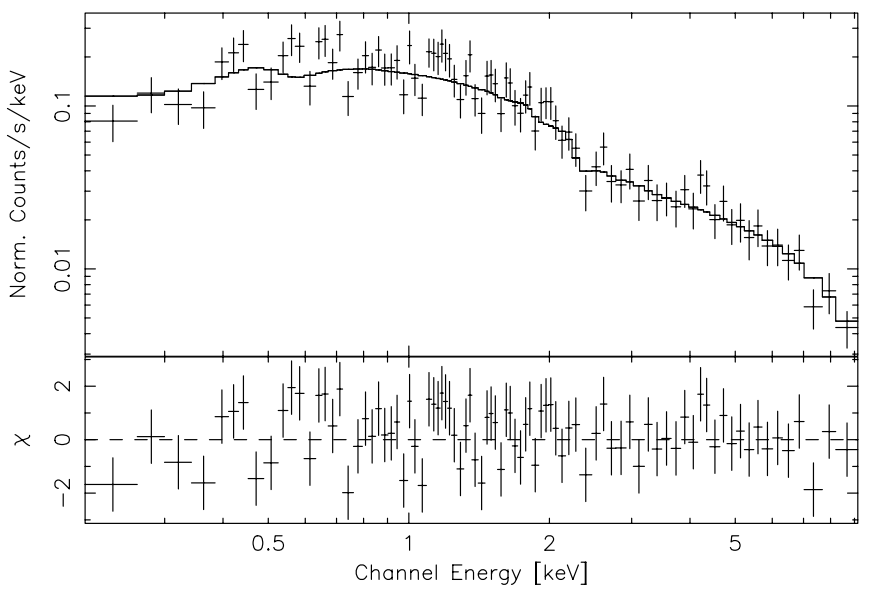

FIG. 2.-Power-law model with Galactic absorption fitted to the PN data of RX J1028.6-0844 $\left(\alpha_{\mathrm{X}}=0.233, N_{\mathrm{H}}=4.59 \times 10^{20} \mathrm{~cm}^{-2}\right.$; Table 1$)$.
1997 and references therein; radio position: $\alpha=10^{\mathrm{h}} 28^{\mathrm{m}} 39^{\mathrm{s}} .0$, $\delta=-08^{\circ} 44^{\prime} 36^{\prime \prime}$ [J2000.0]). The X-ray source position is better determined with the $X M M$-Newton observation to be $\alpha=10^{\mathrm{h}} 28^{\mathrm{m}} 38^{\mathrm{s}} \cdot 9, \delta=-08^{\circ} 44^{\prime} 39^{\prime \prime} \cdot 2(\mathrm{~J} 2000.0)$ for the PN camera. Thus, the difference in radio and X-ray source positions is only 3.2 , well within the $X M M$-Newton absolute pointing accuracy of $15^{\prime \prime}$, and also within the boresight error of $3^{\prime \prime}-4^{\prime \prime}$ (Ehle et al. 2003), making the identification secure.

The radio flux at $5 \mathrm{GHz}$ given in the Parkes Catalogue (Otrupcek \& Wright 1991) is $220 \mathrm{mJy}$. This corresponds to a $K$-corrected luminosity density of $\log l_{4.85 \mathrm{GHz}}=34.6 \mathrm{ergs} \mathrm{s}^{-1}$ $\mathrm{Hz}^{-1}$. The radio-to-optical slope $\alpha_{\text {ro }}{ }^{6}$ was calculated from the $K$-corrected luminosity densities to be $\alpha_{\text {ro }}=0.57$. This converts to a radio loudness $R \approx 950,{ }^{7}$ which makes this source a radio-loud object as per the definition of Kellermann et al. (1989), radio-quiet, -loud division at $R=10$. The radio spectral slope is $\alpha_{r}=-0.3$ (Zickgraf et al. 1997), making this a flat-spectrum, radio-loud object, and the high luminosity suggests a possible BL Lac identification (Yuan et al. 2000). The slope of the X-ray power law of the source is also flat $\left(\alpha_{\mathrm{X}} \approx 0.3\right.$; Table 1), similar to other radio-loud sources (e.g., Elvis el al. 1986; Brinkmann et al. 1997a).

From the $R$ magnitude, $R=18.9 \mathrm{mag}$, given in Zickgraf et al. (1997) at the position of RX J1028-0844, we estimated the rest-frame flux density at $2500 \AA$ to $\log f_{2500 \AA}=-26.9 \mathrm{ergs}$ $\mathrm{s}^{-1} \mathrm{~cm}^{-2} \mathrm{~Hz}^{-1}$, assuming a UV spectral slope $\alpha_{\mathrm{UV}}=0.8$ as given in Vignali et al. (2001, 2003b) and a correction for dereddening with $N_{\mathrm{H}} / E_{B-V}=4.93 \times 10^{21} \mathrm{~cm}^{-2} \mathrm{mag}^{-1}$ (Diplas \& Savage 1994). The unabsorbed rest-frame $2 \mathrm{keV}$ flux density is $\log f_{2 \mathrm{kev}}=-29.0 \mathrm{ergs} \mathrm{s}{ }^{-1} \mathrm{~cm}^{-2} \mathrm{~Hz}^{-1}$, which results in an X-ray loudness of $\alpha_{\mathrm{ox}}=0.86$. This is similar to the value $\alpha_{\text {ox }}=0.79$ given in Vignali et al. (2001) and comparable to the $\alpha_{\text {ox }}$ values of other high-redshift BL Lac sources. The difference in the two values of $\alpha_{\text {ox }}$ is due to the different values of $R$ magnitude used as inputs. We obtained the $R$ magnitude from the USNO A2.0 catalog, while Vignali et al.

\footnotetext{
${ }^{6}$ The radio-to-optical spectral slope is defined by Zamorani et al. (1981) as $\alpha_{\mathrm{ro}}=-0.185 \log \left(l_{2500 \AA} / l_{4.85 \mathrm{GHz}}\right)$

${ }^{7}$ Radio loudness $R=f_{5 \mathrm{GHz}} / f_{4400} \AA$.
} 
TABLE 2

Spectral Fit Parameters of RX J1028.6-0844 for a Power-Law Model with Galactic Neutral Absorption at $z=0$ and Redshifted Intrinsic Absorption

\begin{tabular}{|c|c|c|c|}
\hline Detector & $\begin{array}{c}N_{\mathrm{H}, \text { intr }} \\
\left(10^{22} \mathrm{~cm}^{-2}\right)\end{array}$ & $\alpha_{\mathrm{X}}$ & $\begin{array}{c}\chi^{2} \\
\text { (dof) }\end{array}$ \\
\hline $\mathrm{PN}^{1} \ldots \ldots \ldots$ & $0.781 \pm 0.447$ & $0.300 \pm 0.048$ & $91.0(84)$ \\
\hline MOS $-1^{2}$. & $5.57 \pm 2.68$ & $0.378 \pm 0.105$ & $41.7(35)$ \\
\hline MOS $-2^{2} \ldots \ldots \ldots \ldots \ldots \ldots \ldots$ & $2.17 \pm 2.38$ & $0.302 \pm 0.203$ & $31.4(37)$ \\
\hline MOS $-1^{2}$ and MOS $-2^{2} \ldots \ldots$ & $4.07 \pm 1.83$ & $0.347 \pm 0.075$ & $78.2(75)$ \\
\hline$A S C A$ SIS- $0^{3}$ and SIS- $1^{4} \ldots \ldots \ldots \ldots \ldots \ldots \ldots$ & $17.90 \pm 14.16$ & $0.748 \pm 0.321$ & $24.8(28)$ \\
\hline $\mathrm{PN}^{1}+\mathrm{MOS}-1^{2}$ and MOS $-2^{3} \ldots \ldots \ldots \ldots \ldots \ldots$ & $0.87 \pm 0.41$ & $0.277 \pm 0.036$ & $169.7(160)$ \\
\hline $\mathrm{PN}^{1}+\mathrm{MOS}-1^{2}$ and MOS $-2^{2}+$ & & & \\
\hline$A S C A$ SIS- $0^{3}$ and SIS- $1^{4} \ldots \ldots \ldots \ldots \ldots \ldots$ & $0.89 \pm 0.43$ & $0.280 \pm 0.035$ & 200.7 (189) \\
\hline
\end{tabular}

Note. - The galactic absorption is fixed to $N_{\mathrm{H}, \text { gal }}=4.59 \times 10^{20} \mathrm{~cm}^{-2}$ (Dickey \& Lockman 1990).

1 Energy range $0.2-10 \mathrm{keV}$.

2 Energy range $0.5-7.5 \mathrm{keV}$.

3 Energy range $0.8-7.0 \mathrm{keV}$.

${ }^{4}$ Energy range $0.8-6.5 \mathrm{keV}$.

(2001) used an $R$ magnitude from the literature, probably $R=$ 18.9 given in Zickgraf et al. (1997).

\subsection{BR 0351-1034}

\subsubsection{The XMM-Newton Observation}

The mean count rates are $(6.26 \pm 2.39) \times 10^{-3}$ count $\mathrm{s}^{-1}$ for the EPIC PN, and $(2.93 \pm 0.48)$ and $(2.29 \pm 0.48) \times 10^{-3}$ counts $\mathrm{s}^{-1}$ for the EPIC MOS-1 and MOS-2, respectively. This results in total numbers of background-subtracted source counts of 100 for the PN and 58 and 45 for the MOS- 1 and MOS-2. The number of counts in the PN detector are enough, while those in MOS-1 and MOS-2 are not, to perform a simple spectral analysis. So we focus only on the PN data in the following for spectral analysis.

Table 3 summarizes the results of spectral fits to the EPIC PN data of BR 0351-1034. The first fit was a simple power law with cold absorption of neutral elements in our Galaxy $\left(N_{\mathrm{H}, \mathrm{gal}}=4.08 \times 10^{20} \mathrm{~cm}^{-2}\right.$; Dickey \& Lockman 1990). The fit is good with $\chi^{2}=2.3$ for 7 dof. Figure 4 displays this fit to the PN data of BR 0351-1034. If absorption is allowed to be a free parameter, the best-fit column density is consistent with the Galactic column density within errors. However, given the

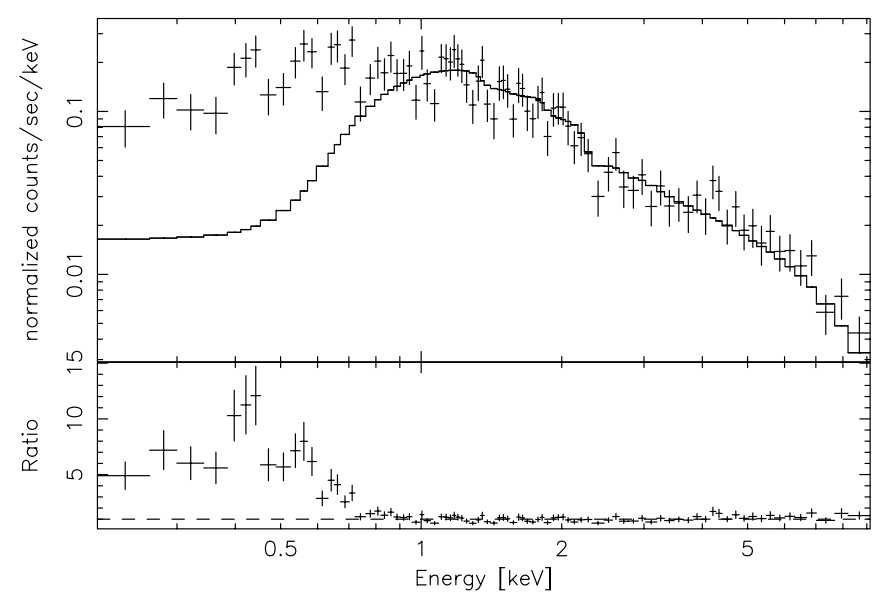

FIG. 3.-Power-law plus neutral Galactic and redshifted intrinsic absorption using the values from the fit to the ASCA data of RX J1028-0844 (Yuan et al. 2000), $N_{\mathrm{H} \text {,intr }}=21.1 \times 10^{22} \mathrm{~cm}^{-2}, \alpha_{\mathrm{X}}=0.72$ ). The figure clearly shows that the $X M M$ data agree with the $A S C A$ model for energies greater than $1 \mathrm{keV}$ but deviate significantly in the soft X-ray band. low data quality, we cannot rule out intrinsic absorption on the order of a few times $10^{22} \mathrm{~cm}^{-2}$ (Table 3, Fig. 5). Motivated by the ROSAT result (see below), we also tried a fit with a broken power-law model with a soft X-ray spectral slope $\alpha_{\mathrm{X} \text {,soft }}=3.5$, as derived from the ROSAT data. The fit is good, implying consistency with the ROSAT data, but the data quality is not high enough to provide a conclusive result on the presence of a broken power law.

\subsubsection{Comparison with ROSAT}

The count rate during the ROSAT PSPC observation was determined using both background regions in the PSPC; both rates agree within the errors with a rate of $3.63 \pm$ $1.3410^{-3}$ counts s$^{-1}$. Based on the PSPC count rate, we expect a $\mathrm{PN}$ count rate $\mathrm{CR}=0.039$ counts $\mathrm{s}^{-1}$ for a simple power-law model with Galactic absorption (see Table 3), but we measured only about one-sixth of it. Clearly the source varied in intensity between the ROSAT and XMM-Newton observations.

The number of photons from the pointed ROSAT PSPC observation was $34.2 \pm 12.7$, not sufficient for a spectral analysis. However, it is possible to derive a hardness ratio ${ }^{8}$ in order to see whether the spectrum is soft or hard. The measured hardness ratio is $\mathrm{HR}=-0.24 \pm 0.57$ and implies a steep soft $\mathrm{X}$-ray spectrum. In order to get a hardness ratio for the PSPC data, a power-law model with Galactic absorption requires a spectral slope $\alpha_{\mathrm{X}}=3.5$. This is a very steep $\mathrm{X}$-ray slope and would be even steeper if the source were also intrinsically absorbed. Thus, it is unlikely that there was excess absorption toward the source during the time of the ROSAT observation. In order to search for spectral variability we used the models derived from the $X M M$ data (Table 3), folded those by the response matrix of the ROSAT PSPC, and calculated the hardness ratios in the PSPC energy band. For a power-law model with $\alpha_{\mathrm{X}}=0.75$ and $N_{\mathrm{H}}=4.08 \times 10^{20} \mathrm{~cm}^{-2}$, we derived an $\mathrm{HR}=+0.51$ and for $\alpha_{\mathrm{X}}=1.19$ and $N_{\mathrm{H}}=13.59 \times$ $10^{20} \mathrm{~cm}^{-2}$, an $\mathrm{HR}=+0.93$. Although this is a rough estimate, it suggests that the source has become harder in the $X M M$ observation compared with the ROSAT PSPC observation $10 \mathrm{yr}$ before (in the observed frame).

Note that a broken power-law model with $\alpha_{\mathrm{X} \text {,soft }}=3.5$ and only Galactic absorption (Table 3) fits the PN data well,

\footnotetext{
${ }^{8} \mathrm{HR}=(H-S) /(H+S)$ with $S$ counts in the $0.1-0.4 \mathrm{keV}$ range and $H=0.5-2.0 \mathrm{keV}$.
} 
TABLE 3

Spectral Fit Parameters to the EPIC PN Data of BR 0351-1034

\begin{tabular}{|c|c|c|c|c|c|}
\hline XSPEC Model & $\begin{array}{c}N_{\mathrm{H}, \mathrm{gal}} \\
\left(10^{20} \mathrm{~cm}^{-2}\right)\end{array}$ & $\begin{array}{c}N_{\mathrm{H}, \text { intr }} \\
\left(10^{22} \mathrm{~cm}^{-2}\right)\end{array}$ & $\alpha_{\mathrm{X} \text {,soft }}$ & $\alpha_{\mathrm{X}, \mathrm{hard}}$ & $\begin{array}{c}\chi^{2} \\
\text { (dof) }\end{array}$ \\
\hline \multirow[t]{2}{*}{ wa power law ${ }^{1} \ldots \ldots \ldots \ldots . . .}$. & $13.59 \pm 12.79$ & $\ldots$ & $\ldots$ & $1.19 \pm 0.66$ & $1.2(4)$ \\
\hline & 4.08 (fixed) & $\ldots$ & $\ldots$ & $0.75 \pm 0.27$ & $2.4(5)$ \\
\hline 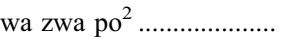 & 4.08 (fixed) & $3.58 \pm 4.45$ & $\ldots$ & $1.08 \pm 0.48$ & $1.1(4)$ \\
\hline \multirow[t]{2}{*}{ wa bknpo ${ }^{3} \ldots \ldots \ldots \ldots \ldots \ldots$} & 4.08 (fixed) & $\ldots$ & 3.50 (fixed) & $0.73 \pm 0.28$ & $3.0(4)$ \\
\hline & $12.70 \pm 11.81$ & $\ldots$ & 3.50 (fixed) & $1.13 \pm 0.62$ & $1.2(3)$ \\
\hline
\end{tabular}

${ }^{1}$ Galactic absorption and power-law model.

${ }^{2}$ Galactic absorption, redshifted neutral absorption at $z=4.351$, and power law.

${ }^{3}$ Galactic absorption and broken power law with observed break energy $E_{\text {break }}=0.45 \pm 0.18 \mathrm{keV}$.

allowing consistency with the ROSAT data. On the other hand, the model with excess absorption brings the expected count rate down by a factor of 3.5 , compared with the observed decrease by a factor of 6 . This means that a variable absorber can be responsible for most of the flux variability observed in BR 0351-1034, but the X-ray source still has to be intrinsically variable by a factor of about 2 .

\subsubsection{Spectral Energy Distribution}

From the UV spectrum given in Storrie-Lombardi et al. (1996b) we derived the optical flux density at the $2500 \AA$ rest frame. The rest-frame flux density at $2 \mathrm{keV}$ was calculated based on the simple power-law fitted to the EPIC PN data (Table 3). This results in an optical-to-X-ray spectral slope $\alpha_{\mathrm{ox}}=1.51$. The $0.2-2.0$ and $2.0-10 \mathrm{keV}$ rest-frame $\mathrm{X}$-ray luminosities are $\log L_{0.2-2.0 \mathrm{keV}}=45.5 \mathrm{ergs} \mathrm{s}^{-1}$ and $\log$ $L_{2.0}-10.0 \mathrm{keV}=46.4 \mathrm{ergs} \mathrm{s}^{-1}$. This converts to a bolometric luminosity of $\log L_{\mathrm{bol}} \approx 46.7 \mathrm{ergs} \mathrm{s}^{-1}$ using the relation ${ }^{9}$ given in Grupe (2004) derived for a low-redshifted, soft $\mathrm{X}$-ray-selected active galactic nucleus (AGN) sample.

\section{DISCUSSION}

\subsection{The Radio-loud QSO RX J1028.6-0844}

As discussed above, RX J1028.6-0844 is a flat-spectrum, radio-loud object. The high radio and X-ray luminosities suggest that the emission is beamed and the source is possibly a BL Lac object, in which we are viewing the source down the radio jet axis. The flat X-ray spectral slope of this source is also consistent with this interpretation. Also, the values of $\alpha_{\mathrm{ox}}$ and $\alpha_{\mathrm{ro}}$ of RX J1028-0844, when placed in the $\alpha_{\text {ox }}$ versus $\alpha_{\text {ro }}$ diagrams of Laurent-Muehleisen et al. (1999) and Beckmann et al. (2003), argue for the BL Lac nature of this source. However, the presence of a $\operatorname{Ly} \alpha$ emission line in the identification spectrum of RX J1028-0844 (Zickgraf et al. 1997) and the lack of significant X-ray variability in the source argue against a blazar classification. Even though a lack of variability is unusual for a BL Lac object, it is not unknown (e.g., Tagliaferri et al. 2003).

Based on ASCA data, RX J1028.6-0844 was believed to have strong intrinsic absorption (§ 3.1.2). Another high-redshift blazar, PMN J0525-3344, also shows extremely strong $\mathrm{X}$-ray absorption, again in an $A S C A$ spectrum, more than a million times greater than the neutral hydrogen or dust column density implied by its optical spectrum (Fabian et al. 2001).

\footnotetext{
9 That is $\log L_{\mathrm{bol}}=0.44+1.02 \times \log L_{0.2-2.0 \mathrm{keV}}$. Note that this relation is determined for a Friedman cosmology with $H_{0}=75 \mathrm{~km} \mathrm{~s}^{-1} \mathrm{Mpc}^{-1}$ and $q_{0}=$ 0.0 and luminosities given in units of watts.
}

Faced with the difficulty of explaining this observation, Fabian et al. (2001) invoke highly ionized and dust-destroyed absorbing gas close to the nucleus, similar to the warm absorber commonly seen in Seyfert galaxies. Another $z>4$ blazar, GB $1428+4217$, was also claimed to have strong intrinsic absorption based on ASCA data (Fabian et al. 1998). Now our $X M M$-Newton observations question these findings, because at least in the case of RX J1028.6-0844 we find the ASCA results to be spurious. As shown in Figure 2 and Table 1 the data are consistent with only Galactic absorption. If present at all, the column density of the intrinsic absorber is more than a factor of 10 smaller than that previously suggested from $A S C A$ observations by Yuan et al. (2000). The superior sensitivity of the XMM EPIC PN detector down to energies of $0.2 \mathrm{keV}$ (Strüder et al. 2001) allows us to put much better constraints on the models than with data of previous X-ray missions.

Our results are in accord with those of Ferrero \& Brinkmann (2003) and Elvis et al. (2000), who also found the evidence for excess absorption in PKS 2149-306 to be marginal, based on XMM-Newton and BeppoSAX data, respectively, in contrast to previous $A S C A$ results (Cappi et al. 1997). It is also interesting to note that ROSAT observations of GB $1428+4217$, which are similarly sensitive to low energies, also imply an order of magnitude lower column density than the ASCA results (Boller et al. 2000). The only radio-loud, high-redshift quasar in which $X M M$ observations confirmed the ASCA claims of excess absorption is PKS 2126-158 (Ferrero \& Brinkmann 2003). Note, however, that PKS 2126-158 is a gigahertz peak spectrum (GPS) source (de Vries, Barthel, \& O'Dea 1997; Stanghellini et al. 1998). GPS

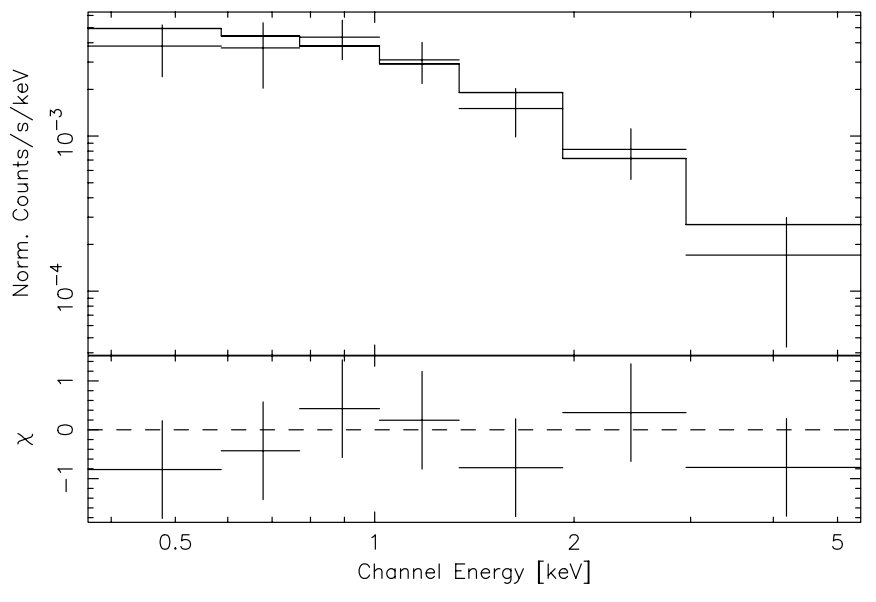

FIG. 4.-Power-law model with neutral Galactic absorption fitted to the EPIC PN data of BR 0351-1034 $\left(\alpha_{\mathrm{X}}=0.75, N_{\mathrm{H}}=4.08 \times 10^{20} \mathrm{~cm}^{-2}\right.$; Table 3). 


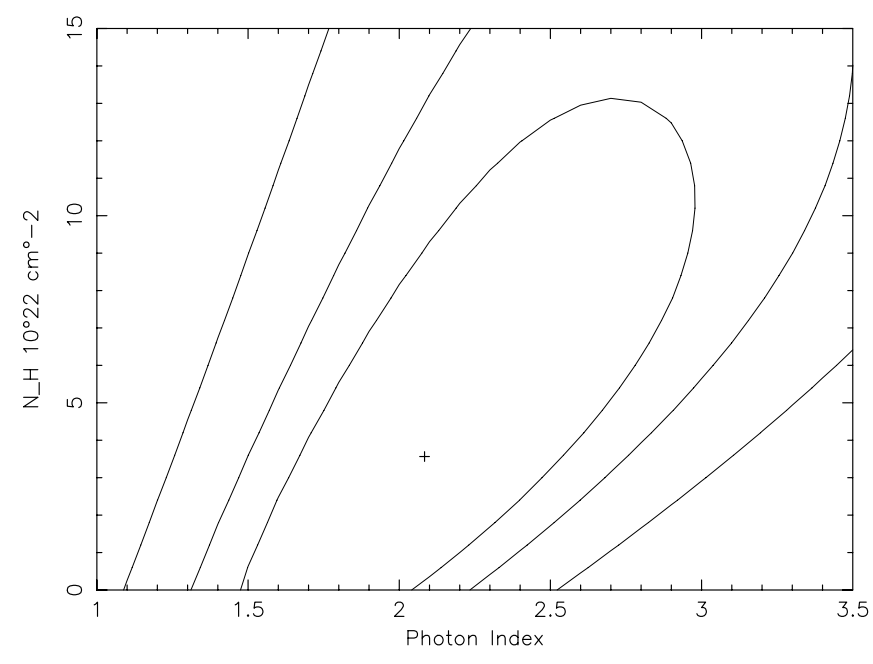

Fig. 5.-Contour plot with the $68 \%, 90 \%$, and $99 \%$ confidence levels of the column density of the intrinsic absorber vs. the photon index of a powerlaw model with Galactic and intrinsic neutral absorption fitted to the EPIC PN data of BR 0351-1034 (Table 3).

sources are thought to be young radio-loud quasars and/or in denser environments (O'Dea, Baum, \& Stanghellini 1991). The excess absorption observed toward PKS 2126-158 may then be in its immediate environment.

However, the claims of excess absorption toward high-redshift, radio-loud quasars are not all from $A S C A$ observations. Elvis et al. (1994) and Cappi et al. (1997) obtained a similar result based on ROSAT observations. It should be noted though that the ROSAT results are not very robust, with excess absorption in candidate radio-loud sources with low-energy cutoffs being consistent with zero within $2 \sigma$ (Fiore et al. 1998). Better quality spectra with XMM-Newton will certainly help resolve this issue, and the effort is already underway.

The excess absorption toward RX J1028.6-0844 is weak, if present at all, and as a result we can neither constrain its metallicity nor redshift. Yuan et al. (2000) have discussed in detail the possibility of intervening material as the absorber and suggested a galaxy about $7^{\prime \prime}$ away from the position of RX J1028.6-0844 found in the image obtained by Zickgraf et al. (1997) as a possible site. Péroux et al. (2001) found a weak damped $\operatorname{Ly} \alpha$ system at $z=3.42$ and $z=4.05$ and estimated the column density to be $\log N_{\mathrm{HI}} \gtrsim 20.1 \mathrm{~cm}^{-2}$. We fitted the $X M M$-Newton PN and MOS data simultaneously to a redshifted absorber with $z=3.42$ and solar abundance resulting in an absorption column $N_{\mathrm{H}}=0.93 \times 10^{22} \mathrm{~cm}^{-2}$. If the abundance is subsolar, similar to that found in other damped Ly $\alpha$ systems (Bechtold et al. 2001), the effective total column density would be even higher. The $z=3.42$ damped system is thus an unlikely site for the X-ray absorption, but a lower redshift damped system is a possibility. It is also quite possible that the excess absorption, if present, is local to our own Galaxy. The observed Galactic column density is averaged over $1 \mathrm{deg}^{2}$ based on the H I maps of Dickey \& Lockman (1990), and it is quite possible that the actual column density is somewhat higher either because of a neutral or molecular cloud.

The major objective of our observing program was to compare the properties of high-redshift quasars to their lowredshift cousins. For this reason, we placed RX J1028.6-0844 on the $\alpha_{\text {ro }}$ versus $\alpha_{\text {ox }}$ plot for complete samples of low-redshift, radio-loud quasars (Fabian et al. 1999; please note that Fabian et al. [1999] define their spectral energy slopes at $5500 \AA$ and $1 \mathrm{keV}$, instead of the conventional $2500 \AA$ and $2 \mathrm{keV}$ ). We find our source to lie right in the region occupied by the BL Lac objects. Comparison with a similar plot by Siebert, Brinkmann, \& Yuan (1998), in which BL Lac objects occupy the regions of $0.6<\alpha_{\mathrm{ox}}<1.6$ and $0.25<\alpha_{\text {ro }}<$ 0.75 , also shows that RX J1028.6-0844 does not occupy any conspicuously different region. Thus, the present observation does not show any evolution of broadband properties with redshift. The $\alpha_{\text {ox }}$ value of RX J1028.6-0844 occupies the lower end of the distribution of radio-loud quasars by Wilkes et al. (1994), implying larger X-ray luminosity, if any, compared with the lower redshift quasars.

\subsection{The Radio-quiet Quasar BR 0351-1034}

BR 0351-1034 is variable by a factor of about 6 on a timescale of $1.75 \mathrm{yr}$ (in the rest frame). While variabilities in X-rays with factors of 3 or 4 on timescales of days are quite common among AGNs (e.g., Leighly 1999; Grupe et al. 2001), evidence of strong variability among high-redshift quasars is sparse. With the present spectral quality, we cannot distinguish among the following scenarios: (1) flux of BR 0351-1034 decreased from the time of ROSAT observation to the time of $X M M$-Newton observations, but the spectrum did not change; (2) flux decreased in part due to excess absorption during the $X M M$-Newton observation; and (3) flux decreased and the spectrum hardened. All three types of variations have been observed in low-redshift AGNs: e.g., in IRAS 1334+24 the flux changed between the RASS and the pointed observation by over a factor of 2, but the spectral shape did not change (Grupe et al. 2001); in NGC 3516 spectral change due to change in absorber column density has been reported (Mathur, Wilkes, \& Aldcroft 1997), and in the narrow-line Seyfert 1 (NLS1) galaxy RX J0134.2-4258 (Grupe et al. 2000) spectral change is observed with change in flux.

The UV/optical-X-ray broadband spectral slope of BR 0351-1024 is $\alpha_{\mathrm{ox}}=1.51$. This is as expected for a radioquiet quasar with optical luminosity density of $\log l_{o}=31.7$ ergs s${ }^{-1} \mathrm{~Hz}^{-1}$ (Yuan et al. (1998); see their Fig. 11). Thus, our observations do not support earlier claims that high-redshift, radio-quiet quasars are weaker in X-rays compared with lower $z$ quasars (Brinkmann et al. 1997b and Ferrero \& Brinkmann 2003).

The most interesting but tentative result is that BR $0351-$ 1024 has a very steep soft X-ray slope. In order to reconstruct the ROSAT PSPC hardness ratio HR $=-0.24$, a steep soft X-ray slope $\alpha_{\mathrm{X}}=3.5$ is needed and the XMM-Newton spectrum is also consistent with such a steep slope ( $\S 3.2 .1)$. While such steep X-ray spectra have been observed in a number of NLS1 galaxies (e.g., Boller, Brandt, \& Fink 1996; Grupe et al. 1998, 2001), only one other high-redshift quasar (SDSS J1044-0125 at $z=5.8$ ) with possible steep X-ray spectrum is known (Mathur 2001; note that the $0.1-0.4 \mathrm{keV}$ range in the observed soft band corresponds to rest frame $0.53-2.1 \mathrm{keV}$ in the rest frame of BR 0351-1034, similar to the ROSAT band). It is commonly believed that the steep soft X-ray spectra in NLS1 galaxies are the result of close to Eddington accretion (e.g., Pounds, Done, \& Osborne 1995). Is BR 0351-1034 also in a very high accretion state? Given the bolometric luminosity of $\log L_{\mathrm{bol}} \approx 46.7 \mathrm{ergs}^{-1}$ the Eddington accretion rate implies a black hole mass of about a 
few times $10^{8} M_{\odot}$. According to accretion-disk corona models (e.g., Kuraszkiewicz et al. 2000), it is difficult to generate such steep X-ray spectra around such massive black holes. Or is the soft X-ray emission a result of Compton thick outflows associated with Eddington or super-Eddington sources (King \& Pounds 2003)? These are intriguing questions, which we can answer only after confirming the suggestive evidence presented here. If true, the steepness of the soft X-ray slope in BR 0351-1034 supports the proposal of Mathur (2000) that high-redshift quasars and NLS1 galaxies are similar objects, perhaps at an early stage of their evolution (Grupe 1996; Grupe et al. 1999; Mathur 2000). This hypothesis has recently been supported by the findings of Yuan \& Wills (2003) who showed that high-redshift quasars are at the same extreme end of the Boroson \& Green (1992) "Eigenvector 1" as the NLS1 galaxies.

\section{SUMMARY \& CONCLUSIONS}

We have studied the XMM-Newton data of the high-redshift blazar RX J1028.6-0844 and the radio-quiet quasar BR 0351-1034. We found that the evidence of excess absorption toward RX J1028.6-0844 is weak at best. If present, the column density of the redshifted absorber is more than 20 times smaller than what has been previously suggested from $A S C A$ data. The location of the absorber is unconstrained, and it may well be in our Galaxy. A longer, $40 \mathrm{ks} X M M-N e w t o n$ observation in $\mathrm{AO} 2$ (PI W. Yuan) will be valuable to confirm the excess absorption if any and to get better constraints on the location and metallicity of the absorber. Our observations of BR 0351-1034 were compromised due to high radiation background, but the present data do not support claims of X-ray weakness in high-redshift, radio-quiet quasars. Similarly, the X-ray properties of RX J1028.6-0844 do not appear to be significantly different from low-redshift BL Lac objects. Clearly, we cannot draw any definite conclusions about quasar evolution from just two observations, and we will publish the results from our entire sample as and when all the observations are made. There is tantalizing evidence of a steep soft X-ray slope in this source, supporting the hypothesis of Mathur (2000) about the evolution of AGNs. We also have more XMM-Newton observations of radio-quiet quasars approved in cycle 2 and 3 , including a longer observation of BR 0351-1034, which will help confirm and extend the results presented here.

We would like to thank Matthias Dietrich for discussions on abundances in high-redshift quasars, Weimin Yuan for intensive discussions on the ASCA observation of RX J1028-0844, Michael Freyberg for discussions on calibration issues, David Weinberg for providing a program to determine the luminosity distances of the sources, Lee Christopher Bassett for comments on the manuscript, and the anonymous referee for a fast referee's report and useful suggestions on the manuscript. This research has made use of the NASA/IPAC Extragalactic Database, which is operated by the Jet Propulsion Laboratory, Caltech, under contract with the National Aeronautics and Space Administration, and data from the US Naval Observatory Catalog A2.0. The ROSAT project is supported by the Bundesministerium für Bildung und Forschung (BMBF/DLR) and the Max Planck Society. This work was supported in part by NASA grant NAG5-9937.
Appenzeller, I., et al. 1998, ApJS, 117, 319

Bechtold, J., et al. 1994, AJ, 108, 374

Bechtold, J., Siemiginowska, A., Aldcroft, T. L., Elvis, M., \& Dobrzycky, A. 2001, ApJ, 562, 133

Beckmann, V., Engels, D., Bade, N., \& Wucknitz, O. 2003, A\&A, 401, 927

Boller, T., Brandt, W. N., \& Fink, H. H. 1996, A\&A, 305, 53

Boller, T., Fabian, A. C., Brandt, W. N., \& Freyberg, M. J. 2000, MNRAS, 315, L23

Boroson, T. A., \& Green, R. F. 1992, ApJS, 80, 109

Brandt, W. N., et al. 2002, ApJ, 569, L5

Brinkmann, W., et al. 1997a, A\&A, 323, 739

Brinkmann, W., Yuan, W., \& Siebert, J. 1997b, A\&A, 319, 413

Cappi, M., Matsuoka, M., Comastri, A., Brinkmann, W., Elvis, M., Palumbo, G. G. C., \& Vignali, C. 1997, ApJ, 478, 492

de Vries, W. H., Barthel, P. D., \& O’Dea, C. P. 1997, A\&A, 321, 105

Dickey, J. M., \& Lockman, F. J. 1990, ARA\&A, 28, 215

Dietrich, M., Appenzeller, I., Vestergaard, M., \& Wagner, S. J. 2002, ApJ, 564, 2002

Diplas, A., \& Savage, B. D. 1994, ApJ, 427, 274

Ehle, M., et al. 2003, XMM-Newton Users' Handbook, Issue 2.1 (Noordwijk: ESA)

Elvis, M., Fiore, F., Siemiginowska, A., Bechtold, J., Mathur, S., \& Mc Dowell, J. 2000, ApJ, 543, 545

Elvis, M., Fiore, F., Wilkes, B. J., \& McDowell, J. 1994, ApJ, 422, 60

Elvis, M., Green, R. F., Bechtold, J., \& Fabbiano, G. 1986, ApJ, 310, 291

Fabian, A. C., Celotti, A., Iwasawa, K., McMahon, R. G., Carili, C. L., Brandt, W. N., Ghisellini, G., \& Hook, I. M. 2001, MNRAS, 323, 373

Fabian, A. C., Celotti, A., Pooley, G., Iwasawa, K., Brandt, W.N., McMahon, R. G., \& Hoenig, M. D. 1999, MNRAS, 308, L6

Fabian, A. C., Iwasawa, K., Celotti, A., Brandt, W. N., McMahon, R. G., \& Hook, I. M. 1998, MNRAS, 295, L25

Ferrero, E., \& Brinkmann, W. 2003, A\&A, 402, 465

Fiore, F., Elvis, M., Giommi, P., \& Padovani, P. 1998, ApJ, 492, 79

Grupe, D. 1996, Ph.D. thesis, Univ. Göttingen 2004, AJ, in press

\section{REFERENCES}

Grupe, D., Beuermann, K., Mannheim, K., \& Thomas, H.-C. 1999, A\&A, 350,805

Grupe, D., Beuermann, K., Thomas, H.-C., \& Fink, H. H. 1998, A\&A, 330,25

Grupe, D., Leighly, K. M., Thomas, H.-C., \& Laurent-Muehleisen, S. A. 2000, A\&A, 356, 11

Grupe, D., Thomas, H.-C., \& Beuermann, K. 2001, A\&A, 367, 470

Haberl, F., Schwope, A. D., Hambaryan, V., Hasinger, G., \& Motch, C. 2003, A\&A, 403, L19

Henry, J. P., et al. 1994, AJ, 107, 1270

Hogg, D. 1999, preprint (astro-ph/9905116)

Irwin, M., McMahon, R. G., \& Hazard, C. 1991, in ASP Conf. Ser. 21, The Space Distribution of Quasars, ed. D. Crampton (San Francisco: ASP), 117

Jansen, F., et al. 2001, A\&A, 365, L1

Kaspi, S., Brandt, W. N., \& Schneider, D. P. 2000, AJ, 119, 2031

Kellermann, K. I., Sramek, R., Schmidt, M., Shaffer, D. B., \& Green, R. 1989, AJ, 98, 1195

King, A. R., \& Pounds, K. A. 2003, MNRAS, 345, 657

Kuraszkiewicz, J., Wilkes, B. J., Czerny, B., \& Mathur, S. 2000, ApJ, 542, 692

Laurent-Muehleisen, S. A., Koolgaard, R. I., Feigelson, E. D., Brinkmann, W., \& Siebert, J. 1999, ApJ, 525, 127

Leighly, K. M. 1999, ApJS, 125, 297

Mathur, S. 2000, MNRAS, 314, L17

- 2001, AJ, 122, 1688

Mathur, S., \& Elvis, M. 1995, AJ, 110, 1551

Mathur, S., Wilkes, B. J., \& Aldcroft, T. 1997, ApJ, 478, 182

Mathur, S., Wilkes, B. J., \& Ghosh, H. 2002, ApJ, 570, L5

O’Dea, C. P., Baum, S. A., \& Stanghellini, C. 1991, ApJ, 380, 66

Otrupcek, R. E., \& Wright, A. E. 1991, Publ. Astron. Soc. Australia, 9, 170

Péroux, C., Storrie-Lombardi, L. J., McMahon, R. G., Irwin, M., \& Hook, I. M. 2001, AJ, 121, 1799

Pfeffermann, E., et al. 1987, Proc. SPIE, 733, 519

Pounds, K. A., Done, C., \& Osborne, J. O. 1995, MNRAS, 277, L5 
Siebert, J., Brinkmann, W., \& Yuan, W. 1998, Aston. Nachr., 319, 1

Stanghellini, C., O’Dea, C. P., Dallacasa, D., Baum, S. A., Fanti, R, \& Fanti, C. 1998, A\&AS, 131, 303

Storrie-Lombardi, L. J., Irwin, M. J., \& McMahon, R. G. 1996a, MNRAS, 282, 1330

Storrie-Lombardi, L. J., McMahon, R. G., Irwin, M. J., \& Hazard, C. 1996b, ApJ, 468, 121

Strüder, L., et al. 2001, A\&A, 365, L18

Tagliaferri, G., et al. 2003, A\&A, 400, 477

Tanaka, Y., Inoue, H., \& Holt, S. S. 1994, PASJ, 46, L37

Tananbaum, H., et al. 1979, ApJ, 234, L9

Trümper, J. 982, Adv. Space Res., 2(4), 241

Turner, M. J. L., et al. 2001, A\&A, 365, L27

Vignali, C., Brandt, W. N., Fan, X., Gunn, J. E., Kaspi, S., Schneider, D. P., \& Strauss, M. A. 2001, AJ, 122, 2155

Vignali, C., Brandt, W. N., \& Schneider, D. 2003a, AJ, 125, 433
Vignali, C., Brandt, W. N., Schneider, D. P., Garmire, G. P., \& Kaspi, S. 2003b, AJ, 125,418

Voges, W., et al. 1999, A\&A, 349, 389

Wilkes, B. J., Tananbaum, H., Worrall, D. M., Avni, Y., Oey, M. S., \& Flanagan, J. 1994, ApJS, 92, 53

York, D. G., et al. 2000, AJ, 120, 1579

Yuan, M. J., \& Wills, B. J. 2003, ApJ, 593, L11

Yuan, W., Brinkmann, W., Siebert, J., \& Wang, W. 1998, A\&A, 330, 108

Yuan, W., Matsuoka, M., Wang, T., Ueno, S., Kubo, H., \& Mihara, T. 2000, ApJ, 545, 625

Zamorani, G., et al. 1981, ApJ, 245, 357

Zickgraf, F.-J., Voges, W., Krauter, J., Thiering, I., Appenzeller, I., Mujica, R., \& Serrano, A. 1997, A\&A, 323, L21

Zimmermann, U., Boese, G., Becker, W., Belloni, S., Döbereiner, S., Izzo, C., Kahabka, P., \& Schwentker, O. 1998, EXSAS User's Guide (MPE Rep.) (Garching: MPI Extraterr. Phys.) 